\title{
On string-localized potentials and gauge fields
}

\author{
Detlev Buchholz ${ }^{1}(\mathbb{D})$ Fabio Ciolli ${ }^{2} \cdot$ Giuseppe Ruzzi $^{2} \cdot$ Ezio Vasselli $^{2}$
}

Received: 2 May 2019 / Revised: 19 August 2019 / Accepted: 21 August 2019 / Published online: 27 August 2019 (c) Springer Nature B.V. 2019

\begin{abstract}
A recent idea, put forward by Mund, Rehren and Schroer, is discussed; it suggests that in gauge quantum field theory, one can replace the point-localized gauge fields by string-localized vector potentials built from gauge-invariant observables and a principle of string independence. Based on a kinematical model, describing unmovable (static) fields carrying opposite charges, it is shown that these string-localized potentials cannot be used for the description of the gauge bridges between electrically charged fields. These bridges are needed in order to ensure the validity of Gauss's law. This observation does not preclude the existence of Poincaré invariant theories, describing the coupling of string-localized gauge-invariant potentials to matter fields. But these potentials are not a full-fledged substitute for the gauge fields in "usual" quantum electrodynamics.
\end{abstract}

Keywords Gauge bridges · String-localized observable potentials · Gauss's law

Mathematics Subject Classification $81 \mathrm{~T} 05 \cdot 83 \mathrm{C} 47 \cdot 57 \mathrm{~T} 15$

\section{Introduction}

It is a well known fact that tensor fields in local quantum field theory, which are closed two-forms on Minkowski space, need not be exact, the most prominent example being the electromagnetic field. One can resolve this cohomological obstruction if one proceeds to indefinite metric extensions of the physical Hilbert space, where the tensor fields can be represented as curls (exterior derivatives) of local vector-valued gauge fields. This fact plays an important role in the perturbative construction of gauge quantum field theories. However, according to folklore, the cohomological difficulties

$\bowtie \quad$ Detlev Buchholz

buchholz@theorie.physik.uni-goettingen.de

1 Mathematisches Institut, Universität Göttingen, Bunsenstr. 3-5, 37073 Göttingen, Germany

2 Dipartimento di Matematica, Universitá di Roma "Tor Vergata", Via della Ricerca Scientifica 1, 00133 Rome, Italy 
can also be resolved within the standard Hilbert space framework of quantum field theory by giving up the locality condition. This can easily be seen by looking in momentum space at the equation, expressing the fact that a field $F$ is a closed twoform (i.e., the homogeneous Maxwell equation),

$$
p_{\gamma} \widetilde{F}_{\alpha \beta}(p)-p_{\beta} \widetilde{F}_{\alpha \gamma}(p)+p_{\alpha} \widetilde{F}_{\beta \gamma}(p)=0
$$

Upon contracting this equation with a fixed vector $e^{\gamma}$ and dividing it (in the sense of distributions) by $e p$, one finds that $F$ can be represented as curl of some non-local vector potential on the original Hilbert space. This field is represented in configuration space by a string-localized integral of $F$.

In recent articles [3-5], it was emphasized by Mund, Rehren and Schroer that the latter field has better ultraviolet properties; this should help to improve the perturbative construction of field theories, where string-localized vector fields, arising from local tensors, are coupled to local matter fields. Since the direction of the chosen vector $e$ ought to be irrelevant for the resulting theory, the authors proposed to solve the renormalization problems arising in the perturbative construction of string-localized fields by a condition of string independence. Indeed, first results seem to indicate that this idea is meaningful [4]. Yet it remained unclear whether the string-localized observable vector fields can replace the local gauge fields appearing in the gauge bridges, connecting electrically charged fields. These gauge bridges are an important ingredient in the construction of the electrically charged superselection sectors.

It is the aim of the present letter to shed light on this problem. For the sake of simplicity and concreteness, we consider the limiting case of quantum electrodynamics, where the gauge field is coupled to static (infinitely heavy, unmovable) fields carrying an electric charge. This assumption allows us to analyze the model at the kinematical (fixed time) level on $\mathbb{R}^{3}$. A convenient gauge in this limit is the so-called $A_{0}=0$ (temporal) gauge. There the spatial components $\boldsymbol{A}, \boldsymbol{E}$ of the vector potential and electric field, respectively, satisfy canonical commutation relations. They can be represented on Hilbert spaces, where the remaining spatial gauge transformations act by unitary operators. The electric field $\boldsymbol{E}$ and magnetic field $\boldsymbol{B} \doteq \operatorname{curl} \boldsymbol{A}$ are gauge invariant and hence are observable. Representations of the observable algebra, describing the electromagnetic field in the absence of charged fields, are characterized by the condition that the charge density $\operatorname{div} \boldsymbol{E}$ vanishes. In representations describing static charges, which are localized at given points in $\mathbb{R}^{3}$, the localization of the observables has to be restricted to the complement of these singularities. Still, the charge density has to vanish in this complement.

The second de Rham cohomology of the punctured space $\mathbb{R}^{3}$ does not vanish, comprising the possible values of the static charges. In spite of the fact that the positions of the static charges are not accessible, it ought to be possible according to Gauss's law to determine their values by surface integrals of the electric field, surrounding their positions; these surface integrals are called flux operators. In order to comply with this condition, opposite charges must be connected by gauge bridges, including the possibility that some bridges extend to spatial infinity if the total charge is different from zero. The gauge bridges are described by automorphisms of the algebra of observables and are constructed from the local vector potential $\boldsymbol{A}$. Their action on flux operators 
which are localized in the spatial complement of the charged fields sharply indicates the value of the charges sitting at the endpoints of the bridges. So the charge content of states can be determined in this manner.

On the other hand, as we shall see, all automorphisms induced by observables act trivially on the flux operators, so they cannot be used to replace the local gauge fields in the gauge bridges. In particular, they do not encode information about the values of the static charges. This holds also true for limits of these automorphisms, obtained, for example, by going to the limiting case of string-localized observable fields. Since none of these automorphisms induces Gauss's law, which is a characteristic of the electric charge, we are led to the conclusion that the string-localized observable vector fields considered in [3-5] do not replace the local gauge fields in every respect.

Our article is organized as follows: In the subsequent section we specify the model, establish our notation and recall some basic cohomological facts. Section 3 contains the definitions of gauge bridges and flux operators and an analysis of their properties. In Sect. 4 the properties of representations containing static charges are discussed and the article closes with some brief conclusions.

\section{The kinematical algebra}

Since we want to study the quantized electromagnetic field in the presence of static (unmovable) charges, we will work at fixed time and consider the so-called temporal $\left(A_{0}=0\right)$ gauge [2]. There the spatial parts $\boldsymbol{A}$ of the vector potential and $\boldsymbol{E}$ of the electric field satisfy canonical commutation relations in Weyl form. Denoting by $\mathcal{D}\left(\mathbb{R}^{3}\right)$ the space of real vector-valued test functions with compact support, the unitary Weyl operators are given by $e^{i \boldsymbol{A}(\boldsymbol{f})}, e^{i \boldsymbol{E}(\boldsymbol{g})}, \boldsymbol{f}, \boldsymbol{g} \in \mathcal{D}\left(\mathbb{R}^{3}\right)$; derivatives of the fields are defined in the sense of distributions. The Weyl operators satisfy the relations

$$
e^{i \boldsymbol{A}(\boldsymbol{f})} e^{i \boldsymbol{E}(\boldsymbol{g})}=e^{i\langle\boldsymbol{f}, \boldsymbol{g}\rangle} e^{i \boldsymbol{E}(\boldsymbol{g})} e^{i \boldsymbol{A}(\boldsymbol{f})},
$$

where $\langle\boldsymbol{f}, \boldsymbol{g}\rangle=\int \mathrm{d} \boldsymbol{x} \boldsymbol{f}(\boldsymbol{x}) \boldsymbol{g}(\boldsymbol{x})$. Weyl operators depending only on the vector potential $\boldsymbol{A}$, or on the electric field $\boldsymbol{E}$, commute among each other and form unitary representations of the vector space $\mathcal{D}\left(\mathbb{R}^{3}\right)$. The full set of Weyl operators generates a $\mathrm{C}^{*}$-algebra $\mathfrak{W}\left(\mathbb{R}^{3}\right)$, the Weyl algebra. It contains for any given open region $\boldsymbol{O} \subset \mathbb{R}^{3}$ a subalgebra $\mathfrak{W}(\boldsymbol{O})$ which is generated by all Weyl operators assigned to test functions $\boldsymbol{f}, \boldsymbol{g} \in \mathcal{D}(\boldsymbol{O})$, the subspace of functions having support in $\boldsymbol{O}$. Operators localized in disjoint regions commute.

On $\mathfrak{W}\left(\mathbb{R}^{3}\right)$ act the spatial gauge transformations $\boldsymbol{A} \mapsto \boldsymbol{A}+\operatorname{grad} \boldsymbol{s}$, where $\boldsymbol{s}$ is any scalar test function. These transformations are induced by the adjoint action of the Weyl operators $e^{i \boldsymbol{E}(\operatorname{grad} s)}=e^{-i(\operatorname{div} \boldsymbol{E})(s)}$. Both, the electric field $\boldsymbol{E}$ and the magnetic field $\boldsymbol{B} \doteq \operatorname{curl} \boldsymbol{A}$ are gauge invariant. They generate the algebra of observables $\mathfrak{A}\left(\mathbb{R}^{3}\right) \subset \mathfrak{W}\left(\mathbb{R}^{3}\right)$. This observable algebra contains for any open region $\boldsymbol{O} \subset \mathbb{R}^{3}$ a local subalgebra $\mathfrak{A}(\boldsymbol{O})$, which is the gauge-invariant part of $\mathfrak{W}(\boldsymbol{O})$. The full algebra $\mathfrak{A}\left(\mathbb{R}^{3}\right)$ can be used if observations are possible everywhere in $\mathbb{R}^{3}$. Yet if static charged fields occupy a given set of spacetime points $X \doteq\left\{\boldsymbol{x}_{1}, \ldots, \boldsymbol{x}_{n}\right\}$, these singular points cannot be accessed. One must then proceed to the subalgebras $\mathfrak{A}\left(\mathbb{R}^{3} \backslash X\right)$. 
Before turning to the analysis of the model, we compile some notation. We will consider integrals of field operators along smooth, simple paths and across closed surfaces, which by definition are the boundary of some region in $\mathbb{R}^{3}$. In order to mollify these integrals, we pick any scalar test function $\boldsymbol{x} \mapsto s(\boldsymbol{x})$, which is normalized according to $\int \mathrm{d} \boldsymbol{x} s(\boldsymbol{x})=1$, and make use of the following definitions of specific test functions.

(i) For given path $\gamma:[0,1] \rightarrow \mathbb{R}^{3}$, we put

$$
\boldsymbol{x} \mapsto \boldsymbol{g}_{\boldsymbol{\gamma}}(\boldsymbol{x}) \doteq \int_{0}^{1} \mathrm{~d} u s(\boldsymbol{x}-\boldsymbol{\gamma}(u)) \dot{\boldsymbol{\gamma}}(u) \in \mathcal{D}\left(\mathbb{R}^{3}\right)
$$

where $\dot{\gamma}$ denotes the tangent vector of $\boldsymbol{\gamma}$.

(ii) For given surface $\sigma:[0,1]^{2} \rightarrow \mathbb{R}^{3}$, we put

$$
\boldsymbol{x} \mapsto \boldsymbol{g}_{\boldsymbol{\sigma}}(\boldsymbol{x}) \doteq \iint_{[0,1]^{2}} \mathrm{~d}^{2} u s(\boldsymbol{x}-\boldsymbol{\sigma}(u)) \boldsymbol{n}_{\boldsymbol{\sigma}}(u) \in \mathcal{D}\left(\mathbb{R}^{3}\right)
$$

where $\boldsymbol{n}_{\boldsymbol{\sigma}}$ is the surface normal and $d^{2} u\left|\boldsymbol{n}_{\sigma}(u)\right|$ the surface form on $\boldsymbol{\sigma}$.

(iii) For given characteristic function $\tau:[0,1]^{3} \rightarrow \mathbb{R}^{3}$, fixing a region, we put

$$
\boldsymbol{x} \mapsto s_{\boldsymbol{\tau}}(\boldsymbol{x}) \doteq \iiint_{[0,1]^{3}} \mathrm{~d}^{3} u s(\boldsymbol{x}-\boldsymbol{\tau}(u)),
$$

which is a scalar test function.

Choosing functions $s$ with sufficiently small support about the origin, the resulting test functions have supports in arbitrarily small neighborhoods of the given regions. For fixed $s$, the following consequences of Gauss's and Stokes' laws are well known, where $\partial$ denotes the boundary of the respective regions.

(i) $\operatorname{grad} s_{\boldsymbol{\tau}}=-\boldsymbol{g}_{\sigma}$, where $\sigma=\partial \boldsymbol{\tau}$

(ii) $\operatorname{curl} \boldsymbol{g}_{\sigma}=-\boldsymbol{g}_{\boldsymbol{\gamma}}$, where $\boldsymbol{\gamma}=\partial \boldsymbol{\sigma}$

(iii) $\operatorname{div} \boldsymbol{g}_{\boldsymbol{\gamma}}=s(\boldsymbol{\gamma}(0))-s(\boldsymbol{\gamma}(1))$.

We also note that $\langle\operatorname{curl} \boldsymbol{f}, \boldsymbol{g}\rangle=\langle\boldsymbol{f}$, curl $\boldsymbol{g}\rangle$ for $\boldsymbol{f}, \boldsymbol{g} \in \mathcal{D}\left(\mathbb{R}^{3}\right)$.

\section{Flux operators and gauge bridges}

Let $X \doteq\left\{\boldsymbol{x}_{1}, \ldots, \boldsymbol{x}_{n}\right\}$ be any given (possibly empty) set of points which are removed from $\mathbb{R}^{3}$. We analyze in this section the properties of Weyl operators which describe flux integrals in $\mathfrak{A}\left(\mathbb{R}^{3} \backslash X\right)$, respectively, gauge bridges between points in $X$.

We begin with the flux integrals. Given any closed surface $\sigma \subset \mathbb{R}^{3} \backslash X$, we pick a corresponding test function $\boldsymbol{g}_{\sigma}$ which also has support in $\mathbb{R}^{3} \backslash \mathcal{X}$. The resulting Weyl operator $e^{i \boldsymbol{E}\left(\boldsymbol{g}_{\sigma}\right)}$ is an element of $\mathfrak{A}\left(\mathbb{R}^{3} \backslash \mathcal{X}\right)$. It commutes with all Weyl operators 
depending only on the electric field $\boldsymbol{E}$. According to the Weyl relations, the commutators with the magnetic field $\boldsymbol{B}=\operatorname{curl} \boldsymbol{A}$ are given by

$$
e^{i \boldsymbol{B}(\boldsymbol{f})} e^{i \boldsymbol{E}\left(\boldsymbol{g}_{\sigma}\right)}=e^{i\left\langle\operatorname{curl} \boldsymbol{f}, \boldsymbol{g}_{\sigma}\right\rangle} e^{i \boldsymbol{E}\left(\boldsymbol{g}_{\sigma}\right)} e^{i \boldsymbol{B}(\boldsymbol{f})}, \quad \boldsymbol{f} \in \mathcal{D}\left(\mathbb{R}^{3} \backslash \mathcal{X}\right) .
$$

Now

$$
\left\langle\operatorname{curl} \boldsymbol{f}, \boldsymbol{g}_{\boldsymbol{\sigma}}\right\rangle=\left\langle\boldsymbol{f}, \operatorname{curl} \boldsymbol{g}_{\boldsymbol{\sigma}}\right\rangle=\left\langle\boldsymbol{f}, \boldsymbol{g}_{\partial \boldsymbol{\sigma}}\right\rangle=0
$$

since $\boldsymbol{\sigma}$ has no boundary, $\partial \boldsymbol{\sigma}=\emptyset$. Thus $e^{i \boldsymbol{E}\left(\boldsymbol{g}_{\sigma}\right)}$ commutes also with all magnetic fields. This leads us to our first result.

Lemma 3.1 Let $\sigma \subset \mathbb{R}^{3} \backslash X$ be a closed surface, and let $\boldsymbol{g}_{\sigma}$ be a corresponding test function, which also has support in $\mathbb{R}^{3} \backslash X$. Then $e^{i \boldsymbol{E}\left(\boldsymbol{g}_{\sigma}\right)}$ is contained in the center of $\mathfrak{A}\left(\mathbb{R}^{3} \backslash \mathcal{X}\right)$. These elements are called flux operators.

Next, we turn to the gauge bridges. These bridges are defined by the adjoint action of Weyl operators, involving the vector potential $\boldsymbol{A}$, on the observables. As a matter of fact, since these bridges connect static charges, we have to proceed to limits of Weyl operators which are no longer elements of the Weyl algebra. But, as we shall see, their adjoint action on the observables is still well defined.

Let $\boldsymbol{x}_{1}, \boldsymbol{x}_{2} \in X$ be a pair of points which is occupied by static charges. For the construction of gauge bridges between these points, we need to exhibit specific test functions. To this end we fix a simple path $\boldsymbol{\gamma}_{0}:[0,1] \rightarrow \mathbb{R}^{3}$ which connects the two points, i.e., $\boldsymbol{\gamma}_{0}(0)=\boldsymbol{x}_{1}, \gamma_{0}(1)=\boldsymbol{x}_{2}$, does not meet other points in $X$, and satisfies

$$
\inf _{u \in(0,1)} u^{-1}(1-u)^{-1}\left|\boldsymbol{\gamma}_{0}(u)-\boldsymbol{x}_{1 / 2}\right| \geq R>0 .
$$

The neighboring paths $\boldsymbol{\gamma}_{\boldsymbol{y}}:[0,1] \rightarrow \mathbb{R}^{3}, \boldsymbol{y} \in \mathbb{R}^{3}$, given by

$$
u \mapsto \boldsymbol{\gamma}_{\boldsymbol{y}}(u) \doteq \boldsymbol{\gamma}_{0}(u)+u(1-u) \boldsymbol{y}, \quad|\boldsymbol{y}|<R,
$$

also connect the two given points and, reducing the value of $R$ if necessary, do not meet any other points in $\mathcal{X}$. Next, we pick some scalar test function $s$ which has support in a ball around the origin of radius $R$ and satisfies $\int \mathrm{d} \boldsymbol{x} s(\boldsymbol{x})=q$ for given $q \in \mathbb{R}$. We then consider for $u \in(0,1)$ the functions

$$
\boldsymbol{x} \mapsto \boldsymbol{a}_{u}(\boldsymbol{x}) \doteq \int \mathrm{d} \boldsymbol{y} s(\boldsymbol{y}) \delta\left(\boldsymbol{x}-\boldsymbol{\gamma}_{\boldsymbol{y}}(u)\right) \dot{\boldsymbol{\gamma}}_{\boldsymbol{y}}(u),
$$

where $\delta$ denotes the Dirac measure. For the chosen paths, these integrals can easily be computed and it is then apparent that the functions $\boldsymbol{a}_{u}$ are test functions having support in $\mathbb{R}^{3} \backslash X$. Moreover their spatial derivatives on compact subsets of $\mathbb{R}^{3} \backslash X$ are uniformly continuous in $u$ and

$$
\boldsymbol{x} \mapsto \operatorname{div} \boldsymbol{a}_{u}(\boldsymbol{x})=-\frac{\mathrm{d}}{\mathrm{d} u} \int \mathrm{d} \boldsymbol{y} s(\boldsymbol{y}) \delta\left(\boldsymbol{x}-\boldsymbol{\gamma}_{\boldsymbol{y}}(u)\right) .
$$

We can proceed now to test functions used for the construction of gauge bridges. Given $\varepsilon>0$, we put 


$$
\boldsymbol{x} \mapsto \boldsymbol{b}_{\varepsilon}(\boldsymbol{x}) \doteq \int_{\varepsilon}^{1-\varepsilon} \mathrm{d} u \boldsymbol{a}_{u}(\boldsymbol{x}) \in \mathcal{D}\left(\mathbb{R}^{3}\right),
$$

so the functions $\boldsymbol{b}_{\varepsilon}$ inherit the support and smoothness properties of their precursors $\boldsymbol{a}_{u}$, and

$$
\lim _{\varepsilon \searrow 0} \operatorname{div} \boldsymbol{b}_{\varepsilon}(\boldsymbol{x})=q \delta\left(\boldsymbol{x}-\boldsymbol{x}_{1}\right)-q \delta\left(\boldsymbol{x}-\boldsymbol{x}_{2}\right)
$$

in the sense of distributions.

After these preparations, we can define automorphisms $\beta_{q, x_{1} x_{2}}(\varepsilon)$ of the Weyl algebra, which approximate a gauge bridge from $\boldsymbol{x}_{1}$ to $\boldsymbol{x}_{2}$ for given charge $q$. They act trivially on Weyl operators depending on $\boldsymbol{A}$ and act on Weyl operators involving the electric field $\boldsymbol{E}$ according to

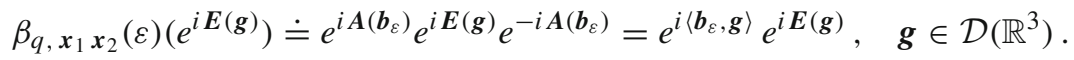

It follows from the preceding discussion that there is some distribution $\boldsymbol{b}_{0}$ such that for any $\boldsymbol{g} \in \mathcal{D}\left(\mathbb{R}^{3}\right)$ and scalar test function $s^{\prime}$ on $\mathbb{R}^{3}$

$$
\lim _{\varepsilon \searrow 0}\left\langle\boldsymbol{b}_{\varepsilon}, \boldsymbol{g}\right\rangle=\left\langle\boldsymbol{b}_{0}, \boldsymbol{g}\right\rangle \text { and } \lim _{\varepsilon \searrow 0}\left\langle\operatorname{div} \boldsymbol{b}_{\varepsilon}, s^{\prime}\right\rangle=q s^{\prime}\left(\boldsymbol{x}_{1}\right)-q s^{\prime}\left(\boldsymbol{x}_{2}\right) .
$$

Since the polynomials of Weyl operators are norm dense in the Weyl algebra, it implies that the automorphisms $\beta_{q, x_{1}} x_{2}(\varepsilon)$, restricted to the observable algebra $\mathfrak{A}\left(\mathbb{R}^{3}\right)$, converge in this limit pointwise in the norm topology to some automorphism $\beta_{q, x_{1} x_{2}}$,

$$
\lim _{\varepsilon \searrow 0} \beta_{q, \boldsymbol{x}_{1} \boldsymbol{x}_{2}}(\varepsilon)(A)=\beta_{q, \boldsymbol{x}_{1} \boldsymbol{x}_{2}}(A), \quad A \in \mathfrak{A}\left(\mathbb{R}^{3}\right) .
$$

This automorphism defines a gauge bridge. Since the underlying test functions $\boldsymbol{b}_{\varepsilon}$ have support in $\mathbb{R}^{3} \backslash \mathcal{X}$, it is apparent that the restriction of $\beta_{q, x_{1} x_{2}}$ to $\mathfrak{A}\left(\mathbb{R}^{3} \backslash \mathcal{X}\right)$ maps this subalgebra onto itself. Some relevant properties of this restriction are compiled in the following lemma.

Lemma 3.2 Let $\beta_{q, x_{1} x_{2}}\left\lceil\mathfrak{A}\left(\mathbb{R}^{3} \backslash \mathcal{X}\right)\right.$ be the restriction of the automorphism defined in equation (3.1). Then

(i) for any scalar test function $s$, having support in $\mathbb{R}^{3} \backslash X$, the operators $e^{i \text { div } \boldsymbol{E}(s)}$ are invariant under the action of $\beta_{q, x_{1}} x_{2}$.

(ii) for any closed surface $\sigma \subset \mathbb{R}^{3} \backslash \mathcal{X}$, the flux operators for the corresponding surface functions $\boldsymbol{b}_{\boldsymbol{\sigma}}$, having support in a sufficiently small neighborhood of $\boldsymbol{\sigma}$, satisfy

$$
\beta_{q, x_{1} \boldsymbol{x}_{2}}\left(e^{i \boldsymbol{E}\left(\boldsymbol{g}_{\sigma}\right)}\right)= \begin{cases}e^{i \boldsymbol{E}\left(\boldsymbol{g}_{\boldsymbol{\sigma}}\right)} & \text { if } \boldsymbol{\sigma} \text { encloses no point in } X \\ e^{i q} e^{i \boldsymbol{E}\left(\boldsymbol{g}_{\sigma}\right)} & \text { if } \boldsymbol{\sigma} \text { encloses only } \boldsymbol{x}_{1} \\ e^{-i q} e^{i \boldsymbol{E}\left(\boldsymbol{g}_{\sigma}\right)} & \text { if } \boldsymbol{\sigma} \text { encloses only } \boldsymbol{x}_{2} \\ e^{i \boldsymbol{E}\left(\boldsymbol{g}_{\boldsymbol{\sigma}}\right)} & \text { if } \boldsymbol{\sigma} \text { encloses, both, } \boldsymbol{x}_{1} \text { and } \boldsymbol{x}_{2} .\end{cases}
$$


(iii) for any relatively compact, contractible region $\boldsymbol{O} \subset \mathbb{R}^{3} \backslash X$ there exists some test function $f_{\boldsymbol{O}} \in \mathcal{D}\left(\mathbb{R}^{3} \backslash \mathcal{X}\right)$ such that

$$
\beta_{q, x_{1} x_{2}}(A)=e^{i \boldsymbol{B}\left(f_{\boldsymbol{O}}\right)} A e^{-i \boldsymbol{B}\left(f_{\boldsymbol{O}}\right)}, \quad A \in \mathfrak{A}(\boldsymbol{O}) .
$$

Proof (i) Recalling that $\operatorname{div} \boldsymbol{E}(s)=-\boldsymbol{E}(\operatorname{grad} s)$, the statement follows from

$$
-\left\langle\boldsymbol{b}_{0}, \operatorname{grad} s\right\rangle=-\lim _{\varepsilon \searrow 0}\left\langle\boldsymbol{b}_{\varepsilon}, \operatorname{grad} s\right\rangle=\lim _{\varepsilon \searrow 0}\left\langle\operatorname{div} \boldsymbol{b}_{\varepsilon}, s\right\rangle=q s\left(\boldsymbol{x}_{2}\right)-q s\left(\boldsymbol{x}_{1}\right)=0 .
$$

(ii) As was mentioned in the preceding section, one has $\boldsymbol{g}_{\sigma}=-\operatorname{grad} s_{\boldsymbol{\tau}}$. Hence

$$
\left\langle\boldsymbol{b}_{0}, \boldsymbol{g}_{\boldsymbol{\sigma}}\right\rangle=-\lim _{\varepsilon \searrow 0}\left\langle\boldsymbol{b}_{\varepsilon}, \operatorname{grad} s_{\boldsymbol{\tau}}\right\rangle=\lim _{\varepsilon \searrow 0}\left\langle\operatorname{div} \boldsymbol{b}_{\varepsilon}, s_{\boldsymbol{\tau}}\right\rangle=q s_{\boldsymbol{\tau}}\left(\boldsymbol{x}_{1}\right)-q s_{\boldsymbol{\tau}}\left(\boldsymbol{x}_{2}\right) .
$$

Since $\boldsymbol{x} \mapsto s_{\tau}(\boldsymbol{x})$ are mollified characteristic functions of the region $\boldsymbol{\tau}$, the statement follows.

(iii) For any scalar test function $s$ with support in $\boldsymbol{O}$, one has

$$
\left\langle\boldsymbol{b}_{0}, \operatorname{grad} s\right\rangle=\lim _{\varepsilon \searrow 0}\left\langle\boldsymbol{b}_{\varepsilon}, \operatorname{grad} s\right\rangle=\lim _{\varepsilon \searrow 0}-\left\langle\operatorname{div} \boldsymbol{b}_{\varepsilon}, s\right\rangle=0
$$

since the points $\boldsymbol{x}_{1}, \boldsymbol{x}_{2}$ lie in the complement of the support of $s$. Since all derivatives of $\boldsymbol{b}_{\varepsilon}$ exist on $\boldsymbol{O}$ and are uniformly continuous in $\varepsilon$, the restriction of the distribution $\boldsymbol{b}_{0}$ to $\mathcal{D}(\boldsymbol{O})$ can be represented by a smooth function $\boldsymbol{b}_{\boldsymbol{O}}$ and $\left\langle\operatorname{div} \boldsymbol{b}_{\boldsymbol{O}}, s\right\rangle=-\left\langle\boldsymbol{b}_{0}, \operatorname{grad} s\right\rangle=0$. Hence $\operatorname{div} \boldsymbol{b}_{\boldsymbol{O}}=0$ on $\boldsymbol{O}$, and since $\boldsymbol{O}$ is contractible, there exists by Poincaré's lemma a smooth function $f_{\boldsymbol{O}}$ such that $\boldsymbol{b}_{\boldsymbol{O}}=\operatorname{curl} \boldsymbol{f}_{\boldsymbol{O}}$. Extending $\boldsymbol{f}_{\boldsymbol{O}}$ to a test function having support in a sufficiently small neighborhood of $\boldsymbol{O}$, it follows that $\left\langle\boldsymbol{b}_{0}, \boldsymbol{g}\right\rangle=\left\langle\operatorname{curl} \boldsymbol{f}_{\boldsymbol{O}}, \boldsymbol{g}\right\rangle, \boldsymbol{g} \in \mathcal{D}(\boldsymbol{O})$. Hence

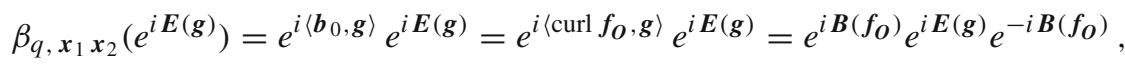

completing the proof.

Having clarified the properties of the flux operators and gauge bridges, we can turn now to the analysis of states.

\section{States containing static charges}

The observable Weyl algebra $\mathfrak{A}\left(\mathbb{R}^{3}\right)$ has an abundance of regular irreducible representations, where the generators of the Weyl operators are densely defined. We pick any such state $\omega_{\emptyset}$ which does not contain charged fields. So the charge density vanishes in this state, i.e., $\omega_{\emptyset}\left(e^{i \operatorname{div} \boldsymbol{E}(s)}\right)=1$ for all scalar test functions $s$ on $\mathbb{R}^{3}$. An example satisfying this condition is the vacuum state of the free electromagnetic field. 
Putting static charges into this state is accomplished by the gauge bridges, defined in the preceding section. We consider here the case of two opposite charges $\pm q$ at given points $X=\left\{\boldsymbol{x}_{1}, \boldsymbol{x}_{2}\right\}$ and briefly comment on more general cases further below. Let $\beta_{q, x_{1} x_{2}}$ be an automorphism of $\mathfrak{A}\left(\mathbb{R}^{3} \backslash \mathcal{X}\right)$, inducing a gauge bridge. We then define a state $\omega_{x}$, containing these charges, by composing $\omega_{\emptyset}$ with this gauge bridge,

$$
\omega \chi(A) \doteq \omega_{\emptyset} \circ \beta_{q, x_{1} x_{2}}(A), \quad A \in \mathfrak{A}\left(\mathbb{R}^{3} \backslash X\right)
$$

The following properties of this state are a direct consequence of Lemma 3.2 and the fact that $\omega_{\emptyset}\left(e^{i \boldsymbol{E}\left(g_{\sigma}\right)}\right)=\omega_{\emptyset}\left(e^{i \operatorname{div} \boldsymbol{E}\left(s_{\boldsymbol{\tau}}\right)}\right)=1$ for all closed surfaces $\boldsymbol{\sigma}$.

Lemma 4.1 Let $\omega x$ be the state defined in equation (4.1). Then

(i) $\omega_{X}\left(e^{i \text { div } \boldsymbol{E}(s)}\right)=1$ for all scalar test functions $s$ having support in $\mathbb{R}^{3} \backslash \mathcal{X}$

(ii) for any closed surface $\sigma \subset \mathbb{R}^{3} \backslash X$ and corresponding surface function $\boldsymbol{g}_{\boldsymbol{\sigma}}$, having support in a sufficiently small neighborhood of $\sigma$, one has

$$
\omega_{x}\left(e^{i \boldsymbol{E}\left(\boldsymbol{g}_{\sigma}\right)}\right)= \begin{cases}1 & \text { if } \boldsymbol{\sigma} \text { encloses none or both points } \boldsymbol{x}_{1}, \boldsymbol{x}_{2} \\ e^{i q} & \text { if } \boldsymbol{\sigma} \text { encloses only } \boldsymbol{x}_{1} \\ e^{-i q} & \text { if } \boldsymbol{\sigma} \text { encloses only } \boldsymbol{x}_{2}\end{cases}
$$

(iii) for any compact, contractible region $\boldsymbol{O} \subset \mathbb{R}^{3} \backslash X$, the restriction $\omega x \uparrow \mathfrak{A}(\boldsymbol{O})$ coincides with a vector state in the GNS representation induced by $\omega_{\emptyset}$.

Part (ii) of this lemma shows that the state $\omega_{x}$ describes a static electric charge $q$ at $\boldsymbol{x}_{1}$ and $-q$ at $\boldsymbol{x}_{2}$, which both can be determined by flux operators (Gauss's law). According to (i), there are no other charges present. Finally, it follows from (iii) that in contractible regions the state cannot be distinguished from states in the superselection sector of $\omega_{\emptyset}$. Let us mention as an aside that these properties prevail in the causal completion of $\mathbb{R}^{3} \backslash X$ in Minkowski space. Going to the full space would require, however, to specify details of the interaction between the field and the charges, which is not necessary here.

Let us turn now to the idea of Mund, Rehren and Schroer to make use of stringlocalized potentials, built from observables. In their approach such operators were concretely given as limits of integrals involving the electromagnetic fields $\boldsymbol{B}, \boldsymbol{E}$. We do not need such detailed information here and will cover arbitrary constructions of this kind, provided they are based on observables. To this end we pick any sequence or, more generally, net of unitary operators $\left\{U_{\iota} \in \mathfrak{A}\left(\mathbb{R}^{3} \backslash X\right)\right\}_{\iota} \in \mathbb{I}$, II being some index set, and consider the corresponding automorphisms

$$
\gamma_{\iota}(A) \doteq U_{\iota} A U_{l}^{-1}, \quad A \in \mathfrak{A}\left(\mathbb{R}^{3} \backslash X\right)
$$

According to standard compactness arguments, any family of states $\left\{\omega_{\emptyset} \circ \gamma_{\iota}\right\}_{\iota \in \mathbb{I}}$ on $\mathfrak{A}\left(\mathbb{R}^{3} \backslash X\right)$ has limit points in the weak-*-topology, which are again states on this algebra. We then have the following result. 
Lemma 4.2 Let $\bar{\omega}$ be any weak-*-limit point of some family $\left\{\omega_{\emptyset} \circ \gamma_{\iota}\right\}_{\iota \in \mathbb{I}}$. Then

$$
\bar{\omega}\left(e^{i \boldsymbol{E}\left(\boldsymbol{g}_{\sigma}\right)}\right)=1
$$

for all closed surfaces $\sigma \subset \mathbb{R}^{3} \backslash X$ and all surface functions $\boldsymbol{g}_{\sigma}$ which have support in a sufficiently small neighborhood of $\sigma$.

Proof According to Lemma 3.1, one has $\gamma_{\iota}\left(e^{i \boldsymbol{E}\left(\boldsymbol{g}_{\sigma}\right)}\right)=U_{\iota} e^{i \boldsymbol{E}\left(\boldsymbol{g}_{\sigma}\right)} U_{\iota}^{-1}=e^{i \boldsymbol{E}\left(\boldsymbol{g}_{\sigma}\right)}$, $\iota \in \mathbb{I}$. Thus

$$
\bar{\omega}\left(e^{i \boldsymbol{E}\left(\boldsymbol{g}_{\sigma}\right)}\right)=\lim _{\iota} \omega_{\emptyset} \circ \gamma_{\iota}\left(e^{i \boldsymbol{E}\left(\boldsymbol{g}_{\sigma}\right)}\right)=\omega_{\emptyset}\left(e^{i \boldsymbol{E}\left(\boldsymbol{g}_{\sigma}\right)}\right) .
$$

But $e^{i \boldsymbol{E}\left(g_{\sigma}\right)}=e^{-i \operatorname{div} \boldsymbol{E}\left(s_{\tau}\right)}$, so the statement follows from the fact that the charge density vanishes everywhere in $\omega_{\emptyset}$.

This lemma shows that there is no way of constructing gauge bridges between charged fields by relying on observable potentials. In particular, it conflicts with the hope that such potentials could be a substitute for the local gauge fields in quantum electrodynamics. In other words, local gauge fields are not only the solutions of cohomological problems, making closed two-forms of observables exact. But, more importantly, they embody fundamental information which is indispensible in physical applications.

We conclude this section by briefly commenting on the structure of states containing several static charges at points $\mathcal{X}=\left\{\boldsymbol{x}_{1}, \ldots, \boldsymbol{x}_{n}\right\}$. Assuming that the values of these charges are $\pm q$, one connects the points $\boldsymbol{x}_{k}, \boldsymbol{x}_{l} \in \mathcal{X}$ by gauge bridges $\beta_{q_{k}, \boldsymbol{x}_{k} \boldsymbol{x}_{l}}$ with variable charges $q_{k} \in \mathbb{R}$. The sum of the charges $q_{k}$ for all paths emanating from a given point $\boldsymbol{x}_{k}$ must be equal to the charge $\pm q$ sitting there. So gauge bridges can connect a given charge with several other charges. One then applies the resulting product of gauge bridges to the state $\omega_{\emptyset}$ in order to obtain a state $\omega_{x}$ for the given configuration $X$. If the total charge of the configuration $X$ is different from zero, one must add to it charges at distant points so that the resulting collection $X_{0} \supset \mathcal{X}$ is neutral. In case of the additional gauge bridges, appearing in this manner, one proceeds to limits, where the added points are shifted to infinity. These limits exist because of the spatial localization properties of the observables. One thereby arrives at consistent models of an arbitrary number of static electric charges in $\mathbb{R}^{3} \backslash X$ for which Gauss's law holds.

\section{Conclusions}

Intrigued by the ideas of Mund, Rehren and Schroer, expounded in [3-5], we have studied in the present article the question whether local gauge fields can be replaced by string-localized potentials, obtained from local observables. Considering the case of the electromagnetic field in the presence of infinitely heavy, electrically charged fields, we have shown that no kind of limiting procedure, involving observable potentials, can approximate the gauge bridges between them. These gauge bridges are an inevitable ingredient of the theory. In particular, they ensure the validity of Gauss's law, i.e., the 
fact that the values of the electric charge can be determined by flux measurements. Thus the gauge bridges describe additional degrees of freedom, sometimes heuristically called "longitudinal photons". They have to be added to the observable string-localized potentials in one way or another.

Our results highlight the fact that the role of gauge fields reaches far beyond the cohomological problem of solving the homogeneous Maxwell equations. This insight prevails in most publications, where it is taken for granted. Even if one is only interested in computations of gauge-invariant operators, the appearance of local gauge fields seems inevitable. For example, the construction of the electric current by Brandt [1] in renormalized perturbation theory, relying on point splitting techniques, involves powers of the gauge fields, compensating gauge degrees of freedom of the charged matter fields in the limit of coinciding points. Similarly, Steinmann in his perturbative construction of cone-localized charged physical fields [6] had to rely on formal power series in terms of local gauge fields. So, together with the present results, we come to the conclusion that the observable string-localized vector potentials, by themselves, are not sufficient to describe all physically relevant features of local gauge fields.

Acknowledgements DB gratefully acknowledges the hospitality and support extended to him by Roberto Longo and the University of Rome "Tor Vergata," which made this collaboration possible. FC and GR are supported by the ERC Advanced Grant 669240 QUEST "Quantum Algebraic Structures and Models." EV is supported in part by OPAL "Consolidate the Foundations." All authors acknowledge support by the MIUR Excellence Department Project, awarded to the Department of Mathematics, University of Rome Tor Vergata, CUP E83C18000100006.

\section{References}

1. Brandt, R.A.: Field equations in quantum electrodynamics. Fortschr. Phys. 18, 249-283 (1970)

2. Löffelholz, J., Morchio, G., Strocchi, F.: Mathematical structure of the temporal gauge in quantum electrodynamics. J. Math. Phys. 44, 5095-5107 (2003)

3. Mund, J.: String-localized quantum fields, modular localization, and gauge theories. In: Sidoravičius, V. (ed.) New Trends in Mathematical Physics, pp. 495-508. Springer, Berlin (2009)

4. Mund, J., Rehren, K.-H., Schroer, B.: Relations between positivity, localization and degrees of freedom: the Weinberg-Witten theorem and the van Dam-Veltman-Zakharov discontinuity. Phys. Lett. B 773, 625-631 (2017)

5. Schroer, B.: An alternative to the gauge theoretic setting. Found. Phys. 41, 1543-1568 (2011)

6. Steinmann, O.: Perturbative Quantum Electrodynamics and Axiomatic Field Theory. Springer, Berlin (2000)

Publisher's Note Springer Nature remains neutral with regard to jurisdictional claims in published maps and institutional affiliations. 